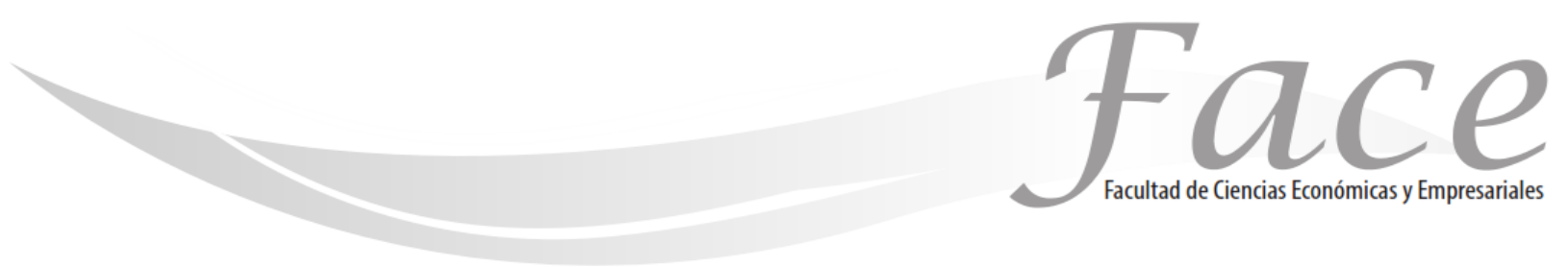

ISSN Impreso: 1794-9920

ISSN Electrónico: 2500-9338

Volumen $18-\mathrm{N}^{\circ} 2$

Año 2018

Págs. 97 - 108

\title{
DIMENSIONES LABORALES DEL SÍNDROME BURNOUT EN DOCENTES INVESTIGADORES DE UNIVERSIDADES PÚBLICAS
}

\author{
Massiel del Carmen Jiménez Ramírez * \\ Enlace ORCID: http://orcid.org/0000-0002-7137-5112 \\ Doile Enrique Ríos-Parra ** \\ Enlace ORCID: http://orcid.org/0000-0002-1974-6924
}

Fecha de Recepción: Julio 22 de 2018

Fecha de Aprobación: Octubre 30 de 2018

\section{Resumen:}

El objetivo del presente estudio se centró en analizar las dimensiones laborales del Síndrome Burnout en docentes investigadores de universidades públicas. Se fundamentó teóricamente en los postulados de Bosqued (2005), Gil - Monte (2005), Maslach y Pines (1977), OIT (2005), Oramas, Hernández, Fernández, (2007), Ortega (2006) y Pagés y Reñé (2008), entre otros. Metodología aplicada fie de tipo descriptiva con diseño no experimental, transeccional y de campo. Se utilizó como técnica la encuesta y como instrumento el cuestionario estandarizado de Maslach Burnout Inventory (MBI) aplicado a 97 docentes investigadores de universidades públicas. Entre los resultados encontrados se corroboró un alto agotamiento emocional con el $40.89 \%$ de las respuestas arrojadas, esto indica que los docentes investigadores casi siempre experimentan cansancio emocional durante el ejercicio de su profesión, incluyendo las actividades complementarias dirigidas al desarrollo de proyectos de investigación. Se concluyó que los indicadores falta de realización personal y despersonalización son bajos mientras que el agotamiento emocional es alto, pues la exigencia al personal docente sobre productos de investigación como garantía de permanencia laboral representa un factor de estrés.

Palabras clave: stress, performance, exhaustion, performance, síndrome

*Universidad Doctor Rafael Belloso Chacín (URBE)-Venezuela, Contacto: mdelcj@gmail.com.

\footnotetext{
** Licenciado en Educación y Licenciado en Filosofía, con títulos de Maestría en Ciencias de la Educación mención Gerencia Educativa, Doctorado en Ciencia de la Educación y Certificación Postdoctoral en Gerencia de la Educación Superior. Contactor: doilerios@gmail.com
} 


\title{
LABOR DIMENSIONS OF THE BURNOUT SYNDROME IN TEACHER-RESEARCHER OF PUBLIC UNIVERSITIES
}

\begin{abstract}
:
The objective of this study was to analyze the labor dimensions of Burnout Syndrome in teachers-researchers from public universities. It was based theoretically on the postulates of Bosqued (2005), Gil - Monte (2005), Maslach and Pines (1977), ILO (2005), Oramas, Hernández, Fernández, (2007), Ortega (2006) and Pagés and Reñé ( 2008), among others. Applied methodology of descriptive type with non experimental, transectional and field design. The survey was used as a technique and as a tool the standardized Maslach Burnout Inventory (MBI) questionnaire applied to 97 research teachers from public universities. Among the results found a high emotional exhaustion was corroborated with the $40.89 \%$ of the answers thrown, this indicates that the investigating teachers almost always experience emotional fatigue during the exercise of their profession, including the complementary activities directed to the development of research projects. It was concluded that the indicators of lack of personal fulfillment and depersonalization are low while emotional exhaustion is high, since the requirement to teaching staff about research products as a guarantee of work permanence represents a stress factor.
\end{abstract}

Keywords: European Data Protection Regulation; Data Protection; Fundamental right; actions; European Union.

\section{DIMENSÕES DO TRABALHO DA SÍNDROME DE BURNOUT NO PROFESSOR-PESQUISADOR DE UNIVERSIDADES PÚBLICAS}

\begin{abstract}
Resumo:
O objetivo deste estudo foi analisar as dimensões do trabalho da Síndrome de Burnout em professores pesquisadores de universidades públicas. Foi baseado teoricamente sobre os postulados da Bosqued (2005), Gil - Monte (2005), Maslach e Pines (1977), da OIT (2005), Oramas Hernandez, Fernandez (2007), Ortega (2006) e Pagés e Rene (2008), entre outros. Metodologia aplicada de tipo descritivo com desenho não experimental, transectional e de campo. A pesquisa foi usada como técnica e como ferramenta para o questionário padronizado do Maslach Burnout Inventory (MBI) aplicado a 97 professores de pesquisa de universidades públicas. Entre os resultados encontrados uma alta exaustão emocional foi corroborada Com a $40,89 \%$ das respostas jogadas, isso indica que os professores quase sempre investigando fadiga emocional experiência Durante o exercício de sua profissão, incluindo as actividades complementares voltadas para o desenvolvimento de projetos de pesquisa. Concluiuse que os indicadores de falta de realização pessoal e despersonalização são baixos, enquanto a exaustão emocional é alta, uma vez que a exigência de pessoal docente sobre produtos de pesquisa como garantia de trabalho continua sendo um fator de estresse.
\end{abstract}

Palavras-chave: estresse, desempenho, exaustão, desempenho, síndrome 


\section{INTRODUCCIÓN:}

A medida que avanza el siglo XXI, los procesos de globalización se van extendiendo e imponiendo; el cambiante mundo de la economía y del trabajo ponen énfasis en controlar y elevar la calidad en la producción, así mismo, los cambios que se vienen produciendo en las sociedades contemporáneas están planteando exigencias importantes de adaptación, y ello a su vez, van introduciendo fuertes cambios en los sistemas de trabajo y la propia actividad laboral.

Todas estas transformaciones y demandas, como la incorporación cada vez más rápida de nuevas tecnologías en el lugar de trabajo, teletrabajo, posibilidades de control y participación en la toma de decisiones, presiones sociales, expectativas, competitividad, los cambios del entorno laboral, tienden a producir una mayor mentalización del trabajo, lo cual puede llevar al individuo hacia un estado de estrés prolongado y agotamiento, afectando su ámbito profesional e integral.

Además, son cada vez mayores las exigencias de expresión emocionales pautadas como parte del desempeño del trabajo, así como, las demandas de iniciativa, innovación y capacidad de adaptación que requieren un aprendizaje permanente y continuo, los cuales influyen sobre las vivencias, comportamiento, respuesta afectivas y emocionales del desempeño laboral.

En este contexto, adquiere relevancia el concepto de trabajo emocional, según Gil-Monte (2005), se puede definir como el acto de expresar emociones socialmente deseadas durante las transacciones en los servicios. Es decir, se espera que los empleados sean capaces de expresar emociones durante las interacciones con los clientes, esta demanda de atención e implicación emocional pudiera afectar la salud mental en los trabajadores. En torno a esto, el autor señala que los trabajadores perteneciente al sector servicio, entre estos los docentes investigadores, pueden ser los más propensos a trastornos depresivos.

Así mismo, el ritmo de vida en la sociedad ha generado un incremento del estrés laboral y los problemas asociados con él. En consecuencia, tal y como lo plantea el mismo autor, uno de cada diez trabajadores de los países industrializados sufre depresión, ansiedad, estrés o cansancio crónico. Señala además, el estrés laboral afecta a casi uno de cada tres trabajadores en la Unión Europea, siendo el segundo problema de salud laboral que más afecta a los trabajadores.

Estas patologías de origen psicosocial inciden en la tasa de accidentes laborales y absentismo disminuyendo el compromiso hacia la organización.

Por otro lado, la Organización Internacional de Trabajo (OIT) (2005), reconoce que el entorno laboral puede ser un agente capaz de deteriorar la salud de las personas durante su desempeño laboral. Del mismo modo, afirma que la salud mental viene determinada en parte por las características del entorno ocupacional, entre estas, los factores psicosociales y organizativos desempeñan un papel relevante.

En este sentido, el ámbito educativo presenta condiciones que pudieran desencadenar factores de índole psicosociales, como por ejemplo: el comportamiento inquieto y ausencia de un plan de incentivos, cambios en la estructura familiar, falta de compromiso por parte de algunos jefes o pares, excesiva de carga de horas directas, falta de recursos materiales. Por otro lado, el bajo salario o estatus socioeconómico e incluso el poco apoyo institucional pueden constituir significativamente fuentes de tensión. Los cuales, tal como lo refiere la OIT, el entorno laboral puede afectar la salud mental, en este caso del docente y su compromiso con la investigación.

Así mismo, se considera que sin educación es imposible alcanzar el desarrollo del individuo y la humanidad, porque ella, le permite al ser humano, integrarse al proceso de socialización, asumir conocimientos, conductas y aptitudes idóneas en pro del bienestar personal y colectivo, aspectos a los cuales cada día se da más importancia en todos los ámbitos sociales.

Igualmente, la mayoría de los profesionales educativos al inicio de su carrera albergan grandes expectativas, así como también, deseos de dejar huellas positivas en la sociedad. Por lo general, estas representan un reto a cumplir debido a las exigencias cada vez mayor de expresiones emocionales pautadas como parte del desempeño del trabajo, y las demandas de iniciativa, innovación y capacidad de adaptación, generando sentimientos de fracaso y frustración, que pueden resultar perjudiciales en la salud del profesorado.

Como se puede inferir, el rol del docente investigador en la sociedad actual es retador, pero a la vez estresante. Del profesor se espera eduque, forme, 
oriente, y también ejerza, en muchas ocasiones, la función de investigador. Estas responsabilidades, junto con las elevadas exigencias cada vez más complejas tanto por parte de estudiantes, jefes de departamentos, como los cambios y reformas de planes de estudio y reestructuración del sistema educativo, pueden estar convirtiendo la docencia en una profesión de alto riesgo.

A este respecto, Oramas, Almirall, Fernández (2007), en una investigación realizada en Venezuela, indican que las causas en la vulnerabilidad del docente a las alteraciones mentales pueden ser amplias y de diversa naturaleza, puesto que, la tarea impone demandas físicas, mentales, emocionales y relacionales que en interacción con los recursos individuales pueden impactar negativamente la salud y bienestar del profesorado, siendo las patologías más frecuentes alteraciones circulatoria, del aparato fonológico y trastornos de salud mental, en especial la ansiedad, e incluso depresión.

En conformidad con esto, el ser humano puede habituarse a circunstancias adversas en momentos difíciles de su vida a través de procesos de adaptación o resiliencia, pero al verse superados éstos, se origina un desborde que ocasiona trastornos psicológicos y orgánicos, pudiendo generar situaciones autodestructivas tanto hacia su persona como para su desempeño dentro de las labores en las que se desenvuelve.

Como se puede apreciar, las condiciones laborales y organizacionales condicionan un ritmo vertiginoso que pudieran afectar el bienestar social y la salud del profesorado. De esta manera, en el ámbito educativo, las obligaciones, exigencias, presiones sociales y profesionales, aumento de responsabilidad y los cambios en las organizaciones generan angustia, agotamiento emocional, trastornos en los ritmos alimenticios, actividad con dolencias físicas, psíquicas y factores de riesgos, poniendo en jaque la salud somática, emocional y conductual de los docentes.

A este respecto, la reciente Ley Orgánica de Prevención, Condiciones y Medio Ambiente de Trabajo (LOPCyMAT, 2005), extiende el concepto de enfermedades ocupacionales, definiéndola como estados patológico contraídos o agravados con ocasión del trabajo o exposición al medio ambiente, las cuales según menciona el artículo 70 , vincula como elementos nuevos, ergonomía, factores psicosociales y emocionales, derivados incluso, de la relación patrono empleador.

En este sentido, la LOPCyMAT, incluye entre los riesgos psicosociales el estrés laboral, el Burnout,
Mobbing. Caracterizándoles como afecciones mentales que a su vez pueden producir trastornos a la salud. Sin embargo, reconoce que las respuestas efectivas para su control y eliminación requieren responsabilidades compartidas, actividades coordinadas de los diferentes actores, es decir, empresas, trabajadores, empleadores, organizados y unidos en pro de la prevención de enfermedades laborales.

Aunado a lo expresado anteriormente, cuando los docentes investigadores sienten que su salud física 0 psíquica da señales evidentes de alarma, entonces se habla del Síndrome Burnout también llamado síndrome de quemarse por el trabajo, de estar quemado o de desgaste profesional y ocurre con frecuencia en los profesionales de organizaciones de servicios que trabajan en contacto directo con personas; es una respuesta al estrés laboral crónico y se considera como la fase avanzada del estrés profesional.

Tal y como lo plantea Rabasa (2007), este síndrome está caracterizado por presentar tres dimensiones claves: agotamiento emocional, actitudes y sentimientos negativos hacia las personas con las que se trabaja y hacia el propio rol profesional (despersonalización), y falta de realización personal en el trabajo, convirtiéndose en un problema de gran relevancia social por las implicaciones que tiene, tanto sobre la vida personal como profesional de los que lo padecen.

Sobre la validez de lo antes planteado, el fenómeno Burnout parece haber aumentado de un tiempo para esta parte, fundamentalmente debido al cambio que se ha operado en la naturaleza misma del trabajo, con lo que su carga mental y emocional se ha visto incrementada. Tanto es así, que en España, el Síndrome Burnout está reconocido como accidente laboral dentro de la profesión docente desde el primero de enero de 2007.

Sin embargo, es importante señalar que diagnosticar docentes que sufren el Síndrome Burnout por encontrarse agotados emocionalmente, sería un error, pues un profesional puede sentir desgaste emocional y no padecer el síndrome. Por lo tanto, un docente puede presentar conductas de apatía, irresponsabilidad e indiferencia con sus compañeros y con la organización pudiendo ser características de su personalidad 0 educación, sin desarrollar Burnout.

Actualmente, en algunas Universidades Públicas del estado Zulia, específicamente en el Municipio Maracaibo, surgió la interrogante ¿cuáles son las dimensiones laborales el Síndrome de Burnout en docentes investigadores de universidades públicas?, pues se pudieron evidenciar condiciones de trabajo 
estresantes, tal vez debido a situaciones intrínsecas, como expectativas de superación, pérdida de la autoestima, pensar que investigar no vale la pena y que no lo valoran, falta de oportunidades de promoción, incidiendo de manera determinante en el bienestar del docente investigador.

Como resultado de lo observado, se refleja que tanto el agotamiento emocional, como el esfuerzo que requiere trabajar con horas directas de catedra, además de otras actividades complementarías; tal vez, debido a la falta de recursos materiales, las condiciones del espacio físico, la cantidad exagerada de matrícula, la desmotivación del profesorado investigador ante la falta de incentivos hacia la investigación, produciendo en el docente una sensación de presión y angustia, reflejando en él sentimientos de culpa y frustración.

Estos y otros síntomas podrían dar origen a un estrés crónico, derivándose una serie de actitudes con signos de apatía, intolerancia, indiferencia, irresponsabilidad, obligándose de esta forma a centrar sus esfuerzos para el cumplimiento de su labor y por lo tanto, produciéndole pérdida de la capacidad para consigo mismo, de esta manera, suele producirse un estado psicológico negativo producido por el Síndrome Burnout.

En efecto, todo esto se traducirá en absentismo laboral, bajas laborales, impuntualidad, falta de calidad de la enseñanza y el aprendizaje de los estudiantes, rechazo a la actividad de investigación para la generación de nuevo conocimiento; lo que origina un deficiente ejercicio de su función como consecuencia de una merma en su salud, lo cual conlleva al fracaso profesional y de las relaciones interpersonales. Todo lo anterior expuesto, conllevó a realizar un estudio para analizar las dimensiones laborales del Síndrome Burnout en docentes investigadores de universidades públicas.

\section{ANTECEDENTES:}

\subsection{Síndrome Burnout}

Antes de dar una definición del Síndrome Burnout, es necesario conocer el desarrollo histórico del concepto. Según Menéndez y Moreno (2006), Burnout, literalmente "estar quemado", es un término anglosajón que se utiliza para denominar a aquel sujeto que se encuentra exhausto y sobrecargado y que ha perdido la ilusión por su trabajo.
Los estudios referentes a este síndrome, se iniciaron en la literatura psicológica en Estados Unidos a mediados de la década de los setenta. Fue, Herbert Freudenberger, quien describió por primera vez este síndrome con un enfoque clínico, al definirlo, como una sensación de fracaso y una experiencia agotadora que resulta de una sobrecarga por exigencias de energía, recursos personales o fuerza espiritual del trabajador. Tomando la definición literalmente del diccionario, con lo que incorpora un término coloquial al lenguaje científico.

Según Pagés y Reñé (2008), el término inglés equivalente burnout era utilizado entre atletas y deportista para aludir a aquel estado en el que la persona no alcanza los resultados esperados de un entrenamiento a fondo. Su categorización y difusión como síndrome se debe principalmente a Malash (1977), quien lo conceptualiza como una respuesta a un estrés emocional crónico caracterizado por agotamiento físico y psicológico, actitud fría y despersonalizada en la relación con los demás y sentimiento de inadecuación a las tareas que se han de desarrollar. Cabe señalar, que la definición anterior es una de la más utilizada por algunos investigadores, porque abarca síntomas que son primordiales o se encuentran presentes en este síndrome, como es el agotamiento físico y emocional, el cual aparece en el profesional que se encuentra bajo un estrés crónico.

A este respecto, Salanova y Llorens (2008), haciendo referencia a los estudios realizados por Maslach, explica que el Síndrome Burnout o Síndrome de estar quemado por el trabajo hace referencia a una metáfora que describe un estado de agotamiento similar a un fuego que se sofoca, una pérdida de energía, una llama que se extingue 0 una batería que se agota. Es de considerar, que el Síndrome Burnout no es lo que coloquialmente se entiende por estar quemado; por el contrario va mucho más allá, tal como lo plantea Gil-Monte (2005), "es un proceso insidioso que tiene consecuencias severas para el individuo". A este respecto, cuando un trabajador dice que está quemado, habitualmente quiere dar a entender que no se encuentra satisfecho en su trabajo, se siente agobiado, está harto 0 a disgusto. Por lo tanto, estos síntomas están más relacionados con los bajos niveles de satisfacción laboral que con la intensidad del Síndrome Burnout.

Ahora bien, durante los últimos años el síndrome Burnout ha sido objeto de multitud de estudios e 
investigaciones, hasta el punto de ser un tópico utilizado en simposios, congresos, mesas redondas, conferencias entre otros eventos; concediéndole un estatus similar a las de otras áreas o temas de estudios relevantes, por consiguiente, lejos de parecer agotado, se proyecta hacia este nuevo siglo dedicándole una atención considerable.

Así pues, para la conceptualización y comprensión del síndrome Burnout, existen una gran variedad de autores, lo cuales se mencionaran a lo largo del estudio, que delimitan su extensión, ayudando a entender qué es y qué no es el término Burnout. Ahora bien, al abordar la definición del Síndrome Burnout, es importante subrayar que esta patología debe ser entendida como una forma de acoso psicosocial en el trabajo.

Desde este punto de vista, en este Síndrome tiene un papel muy importante la presión del trabajo y el ambiente físico que envuelve al profesional en este caso al docente investigador, pues bien, el Burnout es una patología derivada de la interacción del individuo con unas determinadas condiciones psicosociales nocivas de trabajo, las cuales pueden traducirse en un deterioro físico y psíquicos en los docentes, que repercuten en las propias instituciones de educación superior.

A este respecto, Gil-Monte, (2005), lo denomina Síndrome de quemarse por el trabajo, el cual es causado directa y exclusivamente por un riesgo psicosocial del medio ambiente de trabajo, es decir, es la actividad laboral la causante del síndrome y no el trabajador. El autor le da la siguiente definición:

Es un proceso que se desarrolla de manera progresiva debido a la utilización de estrategias de afrontamiento poco funcionales con las que los profesionales intentan autoprotegerse del estrés laboral generado por sus relaciones con los clientes de la organización y, en menor medida, por sus relaciones con la organización. (pág. 44)

Por lo tanto, es una situación en la que el docente investigador se ve desbordado y se percibe impotente para hacer frente a los problemas que genera su entorno laboral, en especial en su ambiente social. En este sentido, y como lo señala el mismo autor, el Síndrome Burnout tiene su origen en el entorno de trabajo, no en el trabajador, de allí que, el problema hay que buscarlo en el medio ambiente laboral, no en el individuo.

Así mismo, los autores Fidalgo, Nogareda, Nogareda, Oncins (2006), concuerdan con Gil-Monte, no solo en denominar al Burnout como Síndrome de quemarse por el trabajo, sino al describirlo como una respuesta, en términos de daños a la salud, derivada de la interacción del individuo con una determinada condiciones picosociales nocivas de trabajo que deberían haberse evaluado y corregido. En consecuencia, los antecedentes previos a la aparición del Burnout están relacionados con la estructura de la organización, lo cual, pueden dar lugar a la aparición de un proceso de estrés crónico.

En este sentido, Esqueda, Escalante, D'Anello, Barreat, D'Orazio (2006), aún cuando lo denominan Síndrome de desgaste Profesional, le añade una característica especial a la conceptualización del Síndrome, ya que, lo define como el resultado de una discrepancia entre los ideales individuales y la realidad de la vida ocupacional diaria. En su forma más extrema, el Burnout representa un punto de ruptura en el cual la capacidad de enfrentarse con el ambiente resulta disminuida y especialmente duro para personas entusiastas e idealistas.

De acuerdo con lo planteado por los autores arriba citados, el Síndrome Burnout llega a ser el resultado final de un proceso de atribución en el cual individuos altamente motivados y comprometidos pierden su espíritu. De esta forma, el Síndrome Burnout puede considerarse como una pérdida progresiva del idealismo, de objetivos y de energía en el trabajador, de esta manera, los docentes que sufren Burnout manifiestan consecuencias negativas tanto para la propia persona como para la institución en la que trabajan.

En consideración con lo antes expuesto, para Bosqued (2005), el Síndrome Burnout afecta con mayor frecuencia a aquellas personas que han elegido su profesión por vocación y que al inicio de su vida laboral se mostraban con mayor entusiasmo, dedicación y entrega, tanto a las tareas propias como a los usuarios de su servicio. Partiendo del supuesto anterior, las personas más vulnerables y las que más posibilidades tienen de padecer los estragos del Síndrome son los profesionales muy exigentes consigo mismos, y perfeccionistas y que en las organizaciones de trabajo cumplen funciones más allá 
de lo que sus organismos, habilidades, talentos y capacidades pueden dar. Esta situación puede ser un arma de doble filo, en vista que brinda satisfacción si se crece profesionalmente, pero también pudiera ocasionar desilusión y apatía si la propia tarea es percibida como intrascendente.

Así mismo, la mayor incidencia del Síndrome Burnout se da en profesionales que ejercen una función asistencial o social, tal como lo refieren Alcover, Martínez, Rodríguez, Domínguez (2007), aquellas ocupaciones que implican trabajar de forma intensiva con personas son las más vulnerables, sin embargo, como lo resaltan los autores, no es el trato con personas lo que provoca la aparición del síndrome, sino la medida en que la interacción con ellas supone una demanda emocional. En el caso de la educación, estos clientes que ejercen una marcada presión desencadenante de Burnout en los docentes son los jefes que exigen como requisito de permanencia en el trabajo la realización y publicación de investigaciones, esto propicia una demanda y requerimiento emocional por parte del profesor.

Por otra parte, tal como lo refiere Gil-Monte (2005), el cansancio o agotamiento emocional es sólo uno de los síntomas del síndrome, por lo tanto, hablar de cansancio emocional como única consecuencia, reduce el síndrome al plano afectivo y emocional del individuo, cuando esta patología es mucho más compleja. Es decir, un docente puede sentirse desgastado emocionalmente sin sufrir el síndrome de Burnout. Por lo tanto, como lo manifiesta la autora arriba señalada, el Síndrome Burnout es un proceso resultante del estrés crónico laboral, por lo tanto, es un estado de agotamiento emocional, mental y físico que proviene de la implicación a largo plazo en situaciones emocionalmente exigentes en las que confluyen expectativas muy elevadas.

En definitiva, se constata que Burnout es un proceso cuya característica esencial es el desgaste psíquico resultado del agotamiento emocional y de patentizar una empatía negativa hacia las personas con las que se trabaja, y hacia el rol que se tiene asignado como investigador, es decir, el docente siente la necesidad de dejar su profesión y, así, no tener que hacer frente a sus responsabilidades y a lo que de él se espera y exige. En suma, el Síndrome Burnout es un resultado al que llegan los docentes investigadores sometidos a malas condiciones de su entorno laboral una vez que han mantenido un estrés prolongado y esto no les ha permitido adaptarse al entorno y por lo tanto, ven frustradas sus expectativas de modificar su situación laboral.

\section{MARCO TEORICO:}

\subsection{Dimensiones laborales}

Para la definición y estudio del constructo Burnout, Gil-Monte (2005), propone que resulta muy beneficioso para evaluar y delimitar el concepto aceptar sólo tres dimensiones: agotamiento emocional, despersonalización y falta de realización personal. Sin embargo, el escritor advierte que al adoptar esta definición se peca de reduccionismo, pues no se recoge el fenómeno en toda su amplitud, se pierde parte de su idiosincrasia y no se evalúan todos los síntomas relevantes.

No obstante, la activada docent comprende varias tareas como, planificación de clase, evaluar a los estudiantes, atender las consultas 0 asesoría, cumplir con normas y procedimientos, participar en los programas de capacitación, así como el de ser investigador. Por tanto, dada la compleja situación en la que éste se encuentra, y en las cuales las demandas laborales y personales escapan de su real capacidad, las posibilidades de desarrollar algunas de las dimensiones del Síndrome Burnout resultan bastantes elevadas.

\section{Agotamiento Emocional}

La actividad docente se caracteriza por realizarse en un ambiente en el que las relaciones interpersonales, interacción social y emocional juegan un papel primordial, las cuales influyen y determinan la práctica pedagógica. En otras palabras, la docencia desde la investigación exige entrega, implicación además de un indudable servicio a los demás.

Por lo cual, vinculado al concepto Burnout, se encuentra el agotamiento emocional, quien para Alcover y otros (2007), representa la manifestación más evidente y la cualidad central 
del Síndrome, refiere el autor que como consecuencia de sobrellevar el exceso de carga emocional la persona experimenta vivencias de cansancio emocional, como si su trabajo les "chupara la sangre" 0 les "vaciara emocionalmente" promoviendo el distanciamiento 0 alejamiento del puesto de trabajo.

Por consiguiente, los autores antes descritos dejan claro que el agotamiento emocional se manifiesta cuando el trabajador toma conciencia de su incapacidad para seguir sirviendo a sus clientes con el mismo entusiasmo como lo hacía en sus primeros tiempos. Sin embargo, Rabasa (2007), puntualiza que la implicación emocional y el deseo de lidiar exitosamente a favor de otros, particularmente cuando tienen problemas, genera una tensión emocional crónica que desemboca en agotamiento emocional.

Visto de esta manera, el agotamiento emocional se considera como la reducción de los propios recursos emocionales y al sentimiento de no tener nada que ofrecer a los demás en el plano afectivo, por lo tanto el profesional ve cómo va disminuyendo la capacidad de entrega hacia los otros, tanto desde el punto de vista psicológico y social. Además, cuando la institución se encuentra en proceso de transformación 0 reingeniería, es posible sea causante de alteraciones emocionales, en palabras de Pereira y Ríos (2017, p.91) "cuando la empresa pasa por un proceso de cambio, es difícil para el personal tener control de sus carreras emocionales". Asimismo, esta inestabilidad de los recursos emocionales propios, es debido al contacto con personas destinatarias de esta prestación en condiciones no ajustadas al trabajador los cuales pueden expresar estar a disgusto, hartos y agobiados con su tarea.

Con respecto al docente investigador, las interacciones intensivas y constantes que se dan en la jornada laboral y los requerimientos emanados de los jefes orientados al indicador investigación exigen una constante implicación y demandas emocionales. Por lo tanto, el rol docente trae consigo una imposición cultural de dedicación y entrega, siempre estar dispuesto para satisfacer las necesidades y carencias afectivas del estudiantado, ésta pudieran generar un agotamiento emocional, pero solamente en la vida laboral, o sea, con el educando y en su acción simultanea de investigar las realidades educativas en las cuales él mismo es sujeto de investigación.

\section{Despersonalización}

Los docentes tienen la responsabilidad de formar integralmente a los sus estudiantes. Sin embargo, la relación docente-estudiante, está enmarcada en circunstancias de extrema ansiedad y aflicción, con demandas de implicación emocional en los problemas tantos de índole pedagógico como personales del educando. De este modo, la salida y mecanismos implicados a esta situación pueden derivar la despersonalización.

En conformidad con lo expuesto por Esqueda y otros (2006), despersonalización es el mecanismo de defensa ante el aumento de sentimientos de impotencia, expresado por pérdida de empatía y cinismo ante las personas objeto del trabajo. En otras palabras, la persona muestra una actitud impersonal y negativa hacia los usuarios. De acuerdo con este postulado, GilMonte (2005), define despersonalización como el desarrollo de actitudes y sentimientos negativos, hacia las personas destinatarias del trabajo, estas, añade el autor, son vistas de forma deshumanizada debido a un endurecimiento afectivo. Incluso, los usuarios del servicio perciben la conducta impersonal del trabajador.

En razón de lo expuesto, despersonalización implica la aparición de sentimientos y actitudes negativas, cínicas e impersonales, además de, aislamiento y etiquetamiento despectivo hacia las personas vinculadas con el trabajo. En tal sentido, este proceso de endurecimiento 0 , incluso, deshumanizado lleva a los profesionales a considerar a las personas merecedoras de sus problemas.

En el contexto educativo, el mecanismo de defensa ante las exigencias emocionales y la interacción constante con los jefes y demás entes educativos pueden generar en el docente una actitud despersonalizada hacia los estudiantes 0 para quienes forman parte del entorno profesional, incluso de resistencia a la actividad investigativa. En consecuencia, la conducta 
despersonalizada y distanciada del docente investigador puede provocar serias dificultades en el proceso educativo: indisciplina, falta de control de la clase, disminución de la seguridad y autoestima, esto a su vez trae consigo resultados deficientes, los cuales son percibidos por quienes lo rodean.

\section{Falta de Realización Personal}

El proceso Burnout supone una interacción de variables afectivas (agotamiento emocional y despersonalización), cognitivas (falta de realización personal en el trabajo) y actitudinales (despersonalización y falta de realización personal), las cuales se articulan entre sí dando paso a la aparición del síndrome. Dicho planteamiento deja ver la relación de variables cognitivas y actitudinales reflejadas en la falta de realización personal. Esta idea es respaldada por Ortega (2006), quien la define como sentimientos de fracaso personal, falta de competencia profesional, incapacidad para afrontar las tensiones, por tanto, disminución de expectativas laborales y de las relaciones interpersonales.

En razón de lo expuesto, es necesario destacar el punto de vista expuesto por Rabasa (2007), para quien esta dimensión pudiera ser la combinación del agotamiento emocional y la despersonalización, añade, que es muy difícil obtener un sentimiento de realización personal cuando uno se siente agotado o hay que ayudar a gente que te resulta indiferente. En tal sentido, la baja realización personal tiene que ver con la falta de recursos relevantes, mientras que el agotamiento y la despersonalización surgen de la presencia de una sobrecarga laboral.

Los aportes anteriores dejan claro que la falta de realización personal, suponen tendencias con respuestas negativas hacia sí mismo y su trabajo, evitación de las relaciones interpersonales, baja productividad, incapacidad para soportar la presión y baja autoestima. Además, se caracteriza por una acusada desilusión e impotencia para dar sentido al trabajo, se experimentan sentimientos de fracaso personal, falta de competencia, esfuerzo, carencias de expectativas laborales y una generalizada insatisfacción. Así pues, en la falta de realización personal, el docente evalúa negativamente su habilidad profesional, se siente mal e insatisfecho con su rol como investigador y tiene una sensación de incompetencia que lo limita y deprime, más aún cree que no mejorará. Esta autoevaluación negativa afecta considerablemente su práctica laboral y las relaciones interpersonales con su entorno.

\section{MATERIALES Y MÉTODOS:}

En atención a lo planteado, el estudio se enmarca en un tipo de investigación descriptiva. De acuerdo con Hernández, Fernández y Baptista (2014), los estudios descriptivos buscan especificar las propiedades, las características y los perfiles importantes de personas, grupos, comunidades o cualquier otro fenómeno que se someta a un análisis. Esto es, decir cómo es y cómo se manifiestan determinados fenómenos, es decir, miden, evalúan 0 recolectan datos sobre diversos aspectos, dimensiones o componentes del estudio a investigar.

De igual manera, según Arias F. (2012), la investigación descriptiva "es la caracterización de un hecho, fenómeno, individuo o grupo, con el fin de establecer su estructura o comportamiento." Los resultados de este tipo de investigación, señala el autor, se ubican en un nivel intermedio en cuanto a la profundidad de los conocimientos se refiere.

En cuanto al diseño de investigación, en este estudio se utilizó un diseño no experimental, transeccional y de campo. A tal fin, Hernández, Fernández y Baptista (2014), señalan que los diseños no experimentales son aquellos que no manipulan las variables de estudio, sino que describen sus propiedades esenciales. De allí su carácter de investigación no experimental, puesto que los investigadores no ejerce control ni manipulación alguna sobre la variable Síndrome Burnout.

Por otra parte, según los autores mencionados, se clasifica en transeccional, cuando se enfocan específicamente a describir la relación entre dos 0 más variables sin alterar su comportamiento en un momento único 0 a través de una sola medición. Por lo tanto, para la investigación y análisis de resultados solo se aplicó el instrumento una sola vez. Asimismo, esta investigación se clasificó como un estudio de campo, obteniendo información directamente en el ambiente institucional objeto de 
estudio; tal como lo expresa Arias (2012), quien indica que los estudios de campo recopilan los datos directamente de la realidad donde ocurren los hechos.

Toda investigación debe plantearse inicialmente la delimitación espacial, es decir, el universo o población en el que se circunscribe la investigación. En este estudio, la población es finita accesible. A este respecto, Arias (2012), señala que una población es

finita cuando se conoce la cantidad de unidades que la integran, asimismo, apunta el autor, que desde el punto de vista estadístico una población finita es la constituida por un número inferior a cien mil unidades. Para el caso, se utilizó la población de 97 docentes investigadores de universidades públicas del municipio Maracaibo del estado Zulia, Venezuela.

En cuanto a la técnica e instrumento utilizados para la recolección de la data, fue la encuesta y el cuestionario, respectivamente. En cuanto a el cuestionario, se ha utilizado el cuestionario de Maslach Burnout Inventory (MBI), un instrumento de prestigio y solidez científica; validado y altamente confiable, reconocido por la sociedad Norteamericana de Psicología, el cual permite determinar el grado de Estrés y Burnout, en función de la intensidad con la que los docentes manifiestan unas determinadas sensaciones.
Los datos se estudiaron a través de la estadística descriptiva, determinando la distribución de las frecuencias y porcentajes en el programa estadístico SPSS; para ello, se realizó un análisis en cada indicador, correspondiente a las dimensiones estudiadas, lo cual se hace, mediante la elaboración de tablas y gráficos, que permitió establecer concordancia con los objetivos propuestos, y en consecuencia, medirla y hacer inferencias de los datos obtenidos.

\section{RESULTADOS:}

El análisis se desarrolla interpretando todas las respuestas obtenidas en el cuestionario aplicado, presentado por indicador, los mismo pueden ser observados en las tablas construidas para tal fin. Se inició el análisis de los datos con la presentación de los resultados de cada variable y su análisis de acuerdo con sus dimensiones. En las tablas se presentan los resultados obtenidos de la aplicación del instrumento, el cual fue aplicado a la población seleccionada para dar respuesta a los objetivos específicos propuestos en esta investigación. A continuación se muestran:

Tabla No. 1

Distribución Porcentual de Dimensiones del Síndrome Burnout

\begin{tabular}{c|c|c|c|c|c}
\hline \hline Alternativas & Nunca & Casi nunca & A veces & Casi siempre & Siempre \\
\hline Población & \multicolumn{5}{|c}{ DOCENTES INVESTIGADORES } \\
\hline Indicador & $\%$ & $\%$ & $\%$ & $\%$ & $\%$ \\
\hline Agotamiento emocional & 4.47 & 3.44 & 18.56 & 40.89 & 32.65 \\
\hline $\begin{array}{c}\text { Despersonalización } \\
\text { Falta de realización } \\
\text { personal }\end{array}$ & 35.05 & 43.99 & 11.68 & 5.15 & 4.47 \\
\hline Porcentaje promedio & 23.48 & 52.58 & 9.28 & 3.09 & 4.47 \\
\hline \hline
\end{tabular}

Fuente: Elaboración propia (2010). 
En la tabla número 1, se exponen los porcentajes de los indicadores relacionados con las dimensiones Síndrome Burnout, en la misma se arrojó el siguiente resultado: el $33.34 \%$ de los docentes investigadores opinaron casi nunca, el $23.48 \%$ nunca, el $16.38 \%$ casi siempre, el 13.86 siempre y el $13.17 \%$ a veces. Resultados que indican que casi nunca se identifican las dimensiones del Burnout en los docentes investigadores de las universidades públicas abordadas.

Asimismo, se hace necesario acotar que el indicador falta de realización personal concentra el porcentaje más alto, de allí que el $52.58 \%$ de los docentes investigadores entrevistados muestren una baja actitud negativa, lo cual quiere decir que los docentes de las instituciones objeto de estudio no consideran excesivas sus expectativas laborales ni tienden a evaluarse de manera negativa.

No obstante, es necesario destacar la evidencia de un alto agotamiento emocional con el $40.89 \%$ de las respuestas arrojadas, esto indica que los docentes investigadores casi siempre experimentan cansancio emocional durante el ejercicio de su profesión, incluyendo las actividades complementarias dirigidas al desarrollo de proyectos de investigación, asimismo también consideran que su actividad laboral le proporcionan estrés.

En este sentido, Gil-Monte (2005), describe el agotamiento emocional, como un agotamiento de energía a los recursos emocionales propios, y una experiencia de estar emocionalmente agotado debido al contacto diario y mantenido con personas a las que hay que atender como objeto de trabajo, en los cuales el sector docente es muy vulnerable.

Ahora bien, los resultados develados no coinciden con lo expuesto por Ortega (2006), quien define la falta de realización personal como sentimientos de fracaso personal, falta de competencia profesional, incapacidad para afrontar las tensiones, por tanto, disminución de expectativas laborales y de las relaciones interpersonales. Sobre las consideraciones realizadas anteriormente, los resultados de esta dimensión develan un comportamiento negativo, es decir, son distintos a lo propuesto por Rabasa (2007); éste considera que el desarrollo de las dimensiones del Síndrome Burnout pueden darse de manera simultáneas, con muestra de abuso, distanciamiento respecto al trabajo y una sensación de ineficacia y falta de realización personal por parte de los docentes en el contexto investigado.

Sin embargo, el autor manifiesta que los docentes desarrollan su actividad laboral en contextos en el que se registran una incidencia significativa de factores de riesgos, esto quiere decir, que ese alto porcentaje de agotamiento emocional no evidencia la presencia del síndrome, pero se constituye en una razón que motiva a tomar acciones para su prevención en la instituciones de educación superior estudiadas.

\section{CONCLUSIONES:}

Tomando en cuenta los resultados alcanzados, producto de la comprobación de las teorías abordadas en la investigación y en función del cumplimiento del objetivo sistematizado, se derivan las siguientes conclusiones:

Al analizar las dimensiones del Síndrome Burnout en docentes investigadores de universidades públicas, efectivamente, se constató que en las instituciones abordadas, los indicadores falta de realización personal y despersonalización son bajos mientras que el agotamiento emocional es alto, pues la exigencia al personal docente sobre productos de investigación como garantía de permanencia laboral representa un factor de estrés.

De allí, que se precisen fortalezas por parte de los docentes investigadores dado que no existe disminución de expectativas laborales ni de las relaciones interpersonales, es decir, el docente mantiene intacto el deseo de superación profesional, incluso de manera colectiva; pero la sobrecarga laboral le impide cumplir con todos los requerimiento, colocando en riesgo la calidad de formación de sus estudiantes, lo cual ha de representar la misión medular de toda casa de estudios.

No se trata de desconocer la necesidad de investigación que requiere el docente en su compromiso de actualización permanente, ni de las responsabilidades académicas en relación a la generación de nuevo conocimiento, incluso de aquel que emerge de su propia práctica pedagógica, sino que, el proceso de construcción investigativa debe representar en ellos (docentes investigadores) un ejercicio ameno, placentero, de crecimiento personal y profesional, y que además, sea reconocido por lo comunidad universitaria.

En consecuencia, se han de diseñar planes de acción que contemplen la consecución de objetivos a partir de la realización de diagnósticos en círculos de acción pedagógica, el cual permita identificar las dimensiones del Síndrome Burnout en los docentes investigadores, a fin de aumentar sus expectativas laborales, incluso promover un programa de incentivos, tales como: 
memorandos de felicitación, espacios de reflexión, encuentros para compartir experiencias, bonificaciones salariales, reconocimientos, viajes, entre otros.

\section{REFERENCIAS:}

Alcover, C., Martínez D., Rodríguez F., Domínguez R., (2007). Introducción a la Psicología del Trabajo. España. Editorial Mc Graw-Hill Interamericana.

Arias F., (2012). El proyecto de Investigación. Editorial Episteme. Venezuela.

Bosqued, M., (2005). ¡Que no te pese el Trabajo! España. Editorial Gestión 2000.

Esqueda, L., Escalante G., D'Anello S., Barreat Y., D'Ozorio A., (2006). Aportes a la psicología social de la salud. Universidad de los Andes. Editorial Venezolana C.A.

Fidalgo M., Nogareda S., Ocins M., (2006). Psicología del Trabajo. Madrid.

Gil - Monte, R., (2005). Síndrome de quemarse por el trabajo. (Burnout): España. Editorial Pirámide.

Hernández R., Fernández C., Baptista P., (2014). Metodología de la Investigación. Editorial Pearson. México.

Ley Orgánica de Prevención, Condiciones y Medio Ambiente de Trabajo. (2005). LOCPCYMAT. Venezuela.

Maslach C. y Pines A. (1977). The burnout syndrome in the day care setting. Child Quarterly, 6, 100-13.
Menéndez, C. y Moreno, F., (2006). Ergonomía para docentes. España. Editorial Graó.

OIT. (2005). Enciclopedia de Salud y Seguridad en el Trabajo. Madrid.

Oramas, A., Hernández, P., Fernández, I., (2007). Estrés Laboral y el Síndrome de Burnout en Docentes Venezolanos. Salud de los Trabajadores. Vol. 15. ISSN 1315-0138.

Ortega M., (2006). Educación para la Salud en el ámbito laboral. Revista Española de Pedagogía. No. 235. España.

Pagés, E., y Reñé, A., (2008). Cómo ser docente y no morir en el intento. Técnicas de concentración y relajación en el aula. España. Editorial Graó.

Pereira, D. J., \& Rios, D. E. (2017). Outplacement como herramienta estratégica para reducir momentos de incertidumbre en organizaciones: una perspectiva reflexiva y atemporal. FACE: Revista de la Facultad de Ciencias Económicas y Empresariales, 17(2), 55-67.

Rabasa, B., (2007). El profesor quemado. El Síndrome Burnout. España. Editorial RiE, Redactors i Editors.

Salanova M., y Llorens S., (2008). Estado actual y retos futuros en el estudio del Burnout. España. 